\title{
Saberes y sabores en diálogo: curricularización de la extensión e Investigación-Acción Participativa
}

\author{
Ana Clara Monteverde \\ Universidad Nacional de Entre Ríos y \\ Universidad de Buenos Aires, Argentina. \\ monteverdeanaclara@yahoo.com.ar
}

Cristina Possidoni

crispossi@gmail.com

\section{Laura Peruzzo}

laura_peruzzo@yahoo.com.ar

RECEPCIÓN: 29/06/18

ACEPTACIÓN FINAL: 20/09/18

\section{Resumen}

"Saberes y sabores en diálogo" tiene como eje la integración de docencia, extensión e investigación desde un diseño de Investigación-Acción Participativa (IAP). Enfrentamos el desafío de diseñar prácticas de extensión integradas al currículo universitario para brindar a los estudiantes la oportunidad de involucrarse en los problemas reales de la comunidad y ejercitar las habilidades propias del investigador. Por ser un proyecto de IAP, se fue construyendo conocimiento a partir de las instancias de trabajo colectivo, que permitieron valorizar los saberes previos de todas las personas ligados a su alimentación, producir recursos educativos que dieron cuenta de las reflexiones generadas durante el proceso y propiciaron el enriquecimiento recíproco. Esta propuesta posibilitó fortalecer y estimular el desarrollo y la formación integral de los futuros profesionales al generar un espacio donde los contenidos curriculares específicos se construyan a la luz de los saberes alimentarios populares, así como estos se entiendan desde el análisis científico.

Palabras clave: curricularización de la extensión, Investigación-Acción Participativa, nutrición, gastronomía.

\author{
Elisa Naef \\ elisafnaef@hotmail.com \\ Universidad Nacional de Entre Ríos, \\ Argentina.
}

Investigación y extensión universitaria / Intervenciones
Knowledge and flavors in dialogue: curricularization of extension and participatory action research

\section{Abstract}

"Knowledge and flavors in dialogue" has as its axis the integration of teaching, extension and research from a participatory action research design. We face the challenge of designing extension practices integrated into the university curriculum, giving students the opportunity to get involved in the real problems of the community by exercising the skills of the researcher. Because it is a participatory action research project, knowledge was built from the collective work instances, which allowed to value the previous knowledge of all people linked to their food, to produce educational resources that gave an account of the reflections generated during the process. reciprocal enrichment. This proposal has allowed to strengthen and stimulate the development and the integral formation of the future professionals generating a space where the specific curricular contents are constructed in the light of the popular alimentary knowledge, as well as these are understood from the scientific analysis.

Keywords: extension curricularization participatory action research, nutrition, gastronomy.
Conhecimento e sabores no diálogo: curricularização da extensão e pesquisa -ação participativa

\section{Resumo}

"Conhecimento e sabores no diálogo" tem como eixo a integração do ensino, extensão e pesquisa a partir de um desenho de pesquisa-ação participativa. Enfrentamos o desafio de projetar práticas de extensão integradas ao currículo universitário, dando aos alunos a oportunidade de se envolverem nos problemas reais da comunidade, exercendo as habilidades do pesquisador. Por se tratar de um projeto de pesquisa-ação participativa, o conhecimento foi construído a partir das instâncias de trabalho coletivo, que permitiram valorizar o conhecimento prévio de todas as pessoas ligadas à sua alimentação, para produzir recursos educacionais que dessem conta das reflexões geradas durante o processo e facilitaram oenriquecimento recíproco. Esta proposta permitiu fortalecer e estimular o desenvolvimento e a formação integral dos futuros profissionais gerando um espaço onde os conteúdos curriculares específicos são construídos à luz do conhecimento alimentar popular,bem como estes entendidos a partir da análise científica.

Palavras-chave: curricularização da extensão, pesquisa-ação participativa, nutrição, gastronomia

Para citación de este artículo: Monteverde, A.C.; Possidoni, C.; Peruzzo, L. y Naef, E. (2018). Saberes y sabores en diálogo: curricularización de la extensión e Investigación-Acción Participativa. +E: Revista de Extensión Universitaria, 8(9), julio-diciembre, 203-219. doi: 10.14409/extension.v8i9. Jul-Dic.7857. 


\section{Introducción}

"Saberes y sabores en diálogo" es un proyecto de extensión que responde a la convocatoria Integración de Funciones Universitarias "Prácticas Integrales \& Territorio 2016" de la Secretaría de Extensión Universitaria y Cultura de la Universidad Nacional de Entre Ríos (UNER). ${ }^{1}$

Tiene como eje la integración de docencia, extensión e investigación, y fue pensado con el desafío de diseñar prácticas de extensión integradas al currículo universitario para brindar a los estudiantes la oportunidad de involucrarse en la resolución de problemas reales de la comunidad y trabajar en escenarios donde el aprendizaje y la enseñanza sean bidireccionales y puedan ejercitarse las habilidades propias que hacen al oficio del investigador.

Participan extensionistas, docentes, investigadores, estudiantes de dos instituciones educativas de la ciudad de Gualeguaychú, Entre Ríos: la Escuela de Educación Técnica N 1 (EET) y la Facultad de Bromatología de la UNER, puntualmente, de 4to., 5to. y 6to. año de la Tecnicatura Superior en Gastronomía, y de 2do. y 3er. año de la Licenciatura en Nutrición.

Para los docentes de las asignaturas participantes, ${ }^{2}$ este proyecto ha implicado problematizar y resignificar la práctica para poner a prueba otras formas de enseñar, aprender y evaluar, siempre orientadas a fortalecer y estimular el desarrollo y la formación integral de los futuros profesionales.

Desde esta perspectiva de integralidad de las funciones universitarias (docencia, extensión e investigación), el proyecto "Saberes y Sabores en diálogo" propone la metodología de Investigación-Acción Participativa (IAP). Este modo de hacer ciencia de lo social es consistente con los objetivos de la extensión ya que permite la construcción colectiva del conocimiento crítico de la realidad, fortalece la capacidad de participación de la comunidad, impulsa la modificación de las condiciones que afectan su vida cotidiana y promueve la apropiación de este conocimiento como instrumento transformador de su realidad.

Las actividades del mencionado proyecto comenzaron en 2016 y continuaron hasta el primer semestre de 2018, buscando promover la creación de un espacio donde los contenidos específicos de las diferentes asignaturas participantes se construyeran a la luz de los saberes alimentarios populares, así como estos se entiendan desde el análisis científico.

El artículo tiene como objetivo presentar la experiencia por el aporte que significa haber podido vincular las tres funciones de la universidad: docencia, extensión e investigación, desde un diseño de IAP.

Este artículo da cuenta de algunos aportes teóricos acerca de la relación entre curricularización y extensión, avanza en las características propias de la IAP que la convierten en el diseño privilegiado para estos abordajes; más adelante presenta la propuesta didáctica de las cátedras intervinientes que enmarcaron cada una de las tareas del proyecto, y finaliza con una exposición de los resultados preliminares y las futuras líneas de acción.

\section{La curricularización y la extensión}

Hablar de extensión universitaria es hablar de una concepción de la universidad como un todo, de una concepción de la sociedad y del país y, fundamentalmente, de un ideal de for- 
mación del profesional universitario y de la persona. En un documento de la UNER del año 1997, Gustavo Menéndez (1997) plantea como impensable un proyecto de universidad que no considere un modelo de sociedad y de país que la contenga.

La concepción reformista de la universidad — base de su inclusión como principio en el '18- se fundamenta en la propuesta política de avanzar en la democratización interna de la universidad, de sus relaciones con la sociedad y en su contribución a la democratización social. Desde este enfoque, se privilegia el trabajo con las mayorías sociales que no acceden a la universidad (Sirvent, 2012).

Según Arocena, la extensión universitaria es entendida

"como el conjunto de actividades de colaboración entre actores universitarios y no universitarios, en formas tales que todos los actores involucrados aportan sus respectivos saberes y aprenden en un proceso interactivo orientado a la expansión de la cultura y a la utilización socialmente valiosa del conocimiento con prioridad a los sectores más postergados". (2011:11)

Estas actividades resultan ser el medio por el cual la comunidad universitaria se vincula con la sociedad en su conjunto a través de actividades propias del mundo universitario que se socializan en forma organizada y responsable. Consiste en una acción social con matriz en la docencia y la investigación, donde se construye conocimiento con acuerdo social (Castro, 2015).

Se considera que la extensión universitaria es una herramienta en la formación de estudiantes y docentes, que suele ser orientadora de la investigación y, eventualmente, puede producir modificaciones en la universidad y en la sociedad (De Michele y Giacomino, 2015).

En línea con Camilloni:

"La participación de los estudiantes en prácticas de extensión habilita aprendizajes que tienen características diversas de las que presentan los que realizan en las actividades convencionales, particularmente porque, en atención a su carácter propio, se desarrollan en situaciones auténticas, esto es, de la vida real, en las que se comprometen en una relación responsable con la comunidad. Los aprendizajes académicos y los aprendizajes profesionales se ponen a prueba en la acción". (2017:61)

La curricularización de la extensión universitaria es una estrategia política apropiada para incorporar las actividades de extensión a la cotidianeidad de la labor universitaria (De Michele y Giacomino, 2015). Supone

"incorporar al trayecto académico (currícula) del estudiante universitario diversas modalidades de acciones de campo fuera del aula, en el territorio, que involucren las tres funciones sustantivas de la educación superior -docencia, investigación y extensión". (Vercellino y Del Carmen, 2014:14)

Adherimos a la concepción de extensión universitaria propuesta por Loustaunau y Rivero (2016), quienes la entienden no solo como promoción cultural sino como planeamiento y ejecución de propuestas de integración/intervención social diversas que se traduzcan en aportes significativos para la formación y desarrollo científico-técnico y que además impliquen un compromiso con el mejoramiento de la sociedad. 


\section{La IAP como diseño privilegiado para la curricularización de la extensión}

El proyecto que dio marco a la presente propuesta de curricularización de la extensión fue un diseño de IAP, el cual es un modo de hacer ciencia de lo social donde se busca que la población involucrada tenga una participación real en el proceso de investigación, es decir, que pueda incidir en las decisiones que tengan que ver con las actividades realizadas en el marco de dicho proceso. Se pretende la objetivación de la realidad en estudio, lo que consiste en el proceso de aprendizaje y de construcción de conocimiento por cuyo intermedio la realidad cotidiana de una población se transforma, tal como la misma población, en objeto de análisis, de estudio y de investigación, por parte de esta. Diversos autores, como Rigal, Sirvent, Lewin, Kemmis, Conteras, caracterizan la IAP como un tipo de diseño que tiene por objetivos construir colectivamente conocimiento crítico sobre dicha realidad, fortalecer la organización social y la capacidad de participación de los sectores populares, promover la modificación de las condiciones que afectan su vida cotidiana, y promover la apropiación de este conocimiento para que sirva de instrumento en la organización de acciones de transformación de la realidad cotidiana.

Las instancias de trabajo que propone este tipo de investigación permiten valorizar los saberes previos de las personas, produce recursos educativos que dan cuenta de las reflexiones generadas durante el proceso y propicia el enriquecimiento recíproco. Se apuesta a la construcción paulatina de maneras diferentes de ver la realidad en el interjuego del conocimiento cotidiano con el conocimiento científico.

Los procesos investigativos y participativos se desenvuelven constituyendo una trama con procesos educativos de aprendizajes individuales y grupales donde el investigador juega un doble rol: el de investigador y el de educador popular (Sirvent y Rigal, 2011).

\section{Propuesta didáctica de las cátedras de Metodología para la curricularización de los contenidos de los aprendizajes}

En las clases de la materia Metodología de la Investigación, cuyos contenidos se curricularizan en este proyecto, se parte de una estrategia didáctica que se encuadra en un enfoque general de trabajo colectivo en torno a cuestiones básicas compartidas por todos los participantes, lo que, a su vez, posibilita que los estudiantes vayan logrando de manera autónoma elaborar las decisiones clave propias del investigador al articular tareas individuales y colectivas realizadas tanto dentro como fuera del aula.

Como docentes, intentamos llevar a la práctica una doble intencionalidad. Por un lado, estimular en los estudiantes la creatividad, libertad, autonomía, pensamiento reflexivo y el compromiso social propios de la ciencia. Por el otro, generar estrategias que les permitan traducir los conceptos vertebrales en procedimientos metodológicos que orienten la toma de decisiones en el proyecto de IAP (Sirvent y Monteverde, 2016)

Procuramos poner en acto los principios de una didáctica fundamentada que concibe la relación teoría y práctica como principio rector de los procesos de enseñanza y aprendizaje (Lucarelli, 2009). En el caso de nuestros espacios de enseñanza, la construcción de cada una de las tareas propias del investigador se convierte en un espacio privilegiado para una práctica de la toma de decisiones del oficio de investigar que articule teoría y empiria. 
Trabajar con las decisiones básicas de un diseño de IAP facilita conocer de qué manera el investigador concibe y busca resolver en la "cocina de investigación" (Sirvent y Rigal, 2017) el "amasado" de teoría y empiria y la relación sujeto/objeto, los que puede ir ajustando a la luz de lo que la realidad manifiesta e impone.

Así, el estudiante empieza a investigar desde el momento en que describe la situación problemática en forma colectiva con los miembros de la comunidad educativa de la EET que será la génesis de la investigación que se lleve a cabo durante los dos años que dura el proyecto. La tarea de tomar decisiones no se reduce solamente a un conjunto de técnicas de trabajo "intelectual", "detrás del escritorio", sino que privilegia la entrada al terreno como nutriente fundamental de las decisiones, de modo de vivenciar ese "amasado" de teoría y empiria que define al oficio del investigador.

A lo largo de las actividades programadas en el presente proyecto, cada estudiante universitario puede integrar y ejercitar los conocimientos adquiridos en la cursada no solo en la cátedra de Metodología de la Investigación sino de otras asignaturas dentro de su disciplina, como Nutrición, Nutrición Materno Infantil, y Técnica y Laboratorio de Dietética, y ponerlos al servicio de la comunidad, viéndose enriquecido también por los conocimientos y prácticas desarrolladas por la población de la EET $N^{\circ} 1$. Las actividades en las que participan los estudiantes de la facultad tienen para ellos un reconocimiento curricular en el cursado de la asignatura Metodología de la Investigación II mediante la entrega de trabajos prácticos, el segundo parcial de la materia y en la presentación de un coloquio al finalizar el ciclo lectivo.

Cada una de las actividades del proyecto permitió especialmente curricularizar contenidos de las asignaturas Metodología de la Investigación de la Licenciatura en Nutrición que se presentan en el apartado siguiente.

\section{Características propias del proyecto de curricularización de la extensión: Saberes y sabores en diálogo}

En el marco del proyecto de IAP, el trabajo conjunto de los estudiantes de la Licenciatura en Nutrición y la comunidad educativa de la EET №1 se presenta como una oportunidad para:

- Reconocer la realidad como fuente privilegiada de posibles problemas a investigar.

- Realizar una experiencia concreta de IAP que les posibilite resignificar, a partir del trabajo empírico, las conceptualizaciones teóricas y vivenciar la "cocina" de la investigación. - Interpretar las culturas como determinantes de la selección, preparación e ingesta de alimentos.

- Construir conocimiento a partir de las instancias de trabajo colectivo con la comunidad que les permita valorizar los saberes previos de todas las personas ligados a su alimentación.

- Producir recursos educativos que den cuenta de las reflexiones generadas durante el proceso. 
- Enriquecerse recíprocamente al complementar sus saberes alimentarios: lo culinario observado desde los conocimientos sobre alimentación saludable y los conocimientos sobre alimentación saludable trabajados desde lo culinario.

- Por ser un diseño de IAP, se privilegia la construcción colectiva (Levy, 2007; Perkins, 2001) del conocimiento a partir de la toma de decisiones de los actores a lo largo de todo el proceso, fortaleciendo la articulación extensión-investigación-docencia. ${ }^{4}$

Se trata de un proyecto de curricularización ${ }^{5}$ que tiene como objetivo promover la creación de un espacio de aprendizaje con otros, significativo y poderoso, y lograr la integración de las distintas asignaturas participantes de la carrera de Licenciatura en Nutrición en un proyecto unificado de diálogo con la comunidad.

Una estrategia puesta en juego para sostener el diálogo constante entre los actores involucrados consistió en la creación de un grupo en Facebook donde se incluyó a todos los actores de ambas instituciones como una forma de comunicación permanente, persiguiendo la participación real del grupo en la toma de decisiones de la investigación y, por lo tanto, en la construcción colectiva del conocimiento.

Las actividades realizadas hasta el momento pueden resumirse en cinco grandes grupos, a saber:

1) Visitas a la EET $N^{\circ} 1$ y reuniones con autoridades y docentes.

Se realizaron durante toda la puesta en marcha del proyecto y tuvieron diferentes objetivos: - En la gestación del proyecto se llevaron a cabo con el fin de lograr la elaboración en conjunto del diseño, teniendo en cuenta los intereses de los actores mencionados. Esas instancias fueron una primera aproximación al ámbito donde se planteó trabajar. - Durante los dos años, se trabajó en conjunto con los docentes acordando y planificando cada siguiente actividad.

- Sobre el final de cada año, los encuentros tuvieron el objetivo de evaluar las actividades realizadas hasta el momento y empezar a planificar las del año siguiente.

- Luego de cada una de estas reuniones, se iban tomando en el aula las decisiones acerca de las acciones a seguir, la planificación de los encuentros individuales y colectivos.

2) Encuentro colectivo "Primeras jornadas de trabajo".

Se efectuaron durante los primeros meses del proyecto y continuaron con la etapa de aproximación y conocimiento de la realidad que enmarcaría las actividades de este proyecto. Estos encuentros se produjeron en la Facultad de Bromatología (UNER) con la participación de los estudiantes secundarios y los de la Licenciatura en Nutrición, acompañados por los docentes de ambas instituciones. E hiceron diversas actividades grupales que incluyeron el análisis de chistes y escenas de películas relacionadas con el acto de cocinar que permitieron la integración y la discusión de algunas problemáticas referidas a

4) La extensión universitaria es uno de los tres pilares de las universidades públicas, junto con la docencia y la investigación.

5) La curricularización consiste en incluir como espacio y/o actividad curricular acreditable actividades de extensión universitaria que supongan el trabajo con miembros de la comunidad (Castro y Oyarbide, 2015). 
la cocina y la alimentación que nos unen como grupo de investigación. Las temáticas que surgieron con fuerza en estos encuentros fueron:

- El análisis de los hábitos alimentarios.

- La presencia de la cultura en la cocina.

- La importancia de trabajar bajo la seguridad e higiene alimentaria.

- La importancia de la innovación en la cocina.

Además, hubo propuestas por parte de los estudiantes, tales como integrar los conocimientos de nutrición y gastronomía "cocinando juntos", trabajar en un proyecto de "información nutricional a la carta" para que los comensales se informen sobre los ingredientes y composición química de cada plato y trabajar con la comunidad de Gualeguaychú.

En 2017 se replicó este encuentro con los alumnos de 4to. año de la EET que ingresaron nuevos al proyecto para considerar también sus ideas previas acerca de la problemática en estudio y conocer lo actuado hasta el momento.

Tanto en estos encuentros colectivos como en los talleres de gastronomía en la EET (descritos en la actividad número 5 , los contenidos metodológicos curricularizados fueron la técnica de observación cualitativa, para la cual se utilizó la técnica del registro a tres columnas, que consiste en considerar los observables en una primera columna, los comentarios del investigador en una segunda, y las categorías emergentes del análisis en una tercera, lo que admite el interjuego entre los significados de los investigadores y los sujetos investigados.

Todos los registros fueron luego analizados haciendo uso del Método Comparativo Constante (Glaser y Strauss, 1967). La teoría fundamentada exige identificar categorías teóricas que son derivadas de los datos recurriendo a la sensibilidad teórica del investigador. Ello exige del investigador comparar contenidos de diversos episodios de entrevistas o de observación con los conceptos teóricos nacientes del esfuerzo de identificar los temas emergentes y, por comparación de los incidentes o unidades de sentido, se analizan las semejanzas y diferencias para encontrar las categorías recurrentes y definir las subcategorías que permiten describir las categorías teóricas que pueden ayudar a comprender el fenómeno en estudio (Glaser y Strauss, 1967; Glaser, 1992).

3) "Encuentros de cocina"

Se realizaron por sugerencia de los estudiantes de Gastronomía y tuvieron lugar en la cocina de la Facultad de Bromatología durante el segundo cuatrimestre del proyecto, con la participación de estudiantes y docentes de ambas instituciones organizados por comisiones.

En cada encuentro, la actividad principal fue elaborar juntos un plato propuesto por los estudiantes de Gastronomía y analizar su composición nutricional en el marco de los contenidos curriculares de la cátedra de Nutrición de la Licenciatura en Nutrición.

Además, se debatió, a partir de una presentación, sobre el nexo enriquecedor entre gastronomía y nutrición y sobre los diferentes criterios que tienen en cuenta los nutricionistas y gastronómicos al momento de pensar un plato, por ejemplo:

- La aplicación de diversas técnicas de cocción.

- La búsqueda de variedad de preparaciones.

- El interés por realizar la presentación de los platos. 
- El uso de los utensilios.

- La consideración del tamaño de las porciones.

- La consideración del valor calórico de los platos.

- El control de las pautas de seguridad e higiene en la cocina.

- La posibilidad de encontrar un equilibrio entre calidad nutricional, sabor y presentación.

4) Encuentros de trabajo individual con los alumnos de la EET.

Se llevaron adelante dos instancias específicas de trabajo individual con los alumnos.

La primera, en los hogares de los alumnos que fueron seleccionados como muestra intencional en la toma de entrevistas en profundidad para ahondar en las categorías teóricas surgidas del análisis de los registros de los diversos encuentros colectivos de trabajo. En esta ocasión, los alumnos de Nutrición pudieron vivenciar la implicancia que caracteriza el modo de hacer ciencia cualitativo, el encuentro con el otro y la doble hermenéutica en la construcción de significados (Giddens, 1995). Con este concepto, el autor da cuenta de la imbricación, de la interpenetración que existe entre el saber científico y el saber de sentido común, en la cual el conocimiento lego no contamina sino que debe ser parte de las explicaciones científicas sobre lo social.

En estos encuentros, la técnica privilegiada como contenido a curricularizar fue la entrevista en profundidad. De este diálogo surgieron las categorías construidas que permitieron conocer los saberes de los estudiantes de Gastronomía para seguir trabajando en la comprensión de los posibles nexos entre gastronomía y nutrición.

A partir de las categorías surgidas de las entrevistas, a fin de ampliar la muestra y permitir la generalización de los resultados, se definieron variables para medir mediante encuestas que se sometieron a un análisis estadístico. Los resultados de estos análisis cualitativos y cuantitativos se presentan más adelante en el apartado "Resultados preliminares".

La segunda instancia tuvo lugar en la escuela y consistió en la toma de una encuesta a todo el universo de alumnos participantes del proyecto para medir, desde un abordaje cuantitativo, las variables operacionalizadas a partir de las categorías teóricas derivadas de la etapa cualitativa.

Los alumnos de nutrición vivenciaron todo el proceso cuantitativo de toma de decisiones con relación a la técnica de encuesta: operacionalización de las variables, construcción del instrumento y análisis estadístico a partir de los contenidos trabajados en la materia Bioestadística.

5) Observación de los talleres de gastronomía en la EET

Esta etapa se está llevando a cabo en este momento como segunda actividad de 2017. Los alumnos de 3er. año de Nutrición están haciendo registros de observación del trabajo cotidiano de los alumnos de Gastronomía y sus docentes en los talleres semanales donde realizan la práctica de cocina y ponen en juego los aprendizajes teóricos que trabajan durante la semana.

Los alumnos de 2do. año de Nutrición están trabajando junto a los alumnos de la EET la composición nutricional de las preparaciones, que fue otra de las demandas de los grupos el año pasado. 


\section{Resultados preliminares del proyecto "Saberes y sabores en diálogo"}

\section{Primeras categorías analizadas en las entrevistas}

El análisis de las entrevistas a los estudiantes de Gastronomía dio lugar a la construcción de cuatro categorías principales que se resumen a continuación.

\section{Familia y cocina}

En las entrevistas surgió con fuerza el tema de la comensalidad ligado a la familia, es decir, la forma en que los alimentos se comparten. El inicio del gusto por la cocina, los primeros acercamientos de los estudiantes con la gastronomía, también se observaron ligados a la familia, a la enseñanza de algún familiar, al compartir el acto de cocinar con los miembros de la familia. También se destacó la importancia de la transmisión de saberes culinarios de generación en generación: al cocinar en familia, además de compartir momentos, se aprenden saberes de generaciones anteriores, se intercambian ideas y, junto con lo que ellos aprenden en el colegio, se generan nuevas versiones de recetas que se transmitirán a las siguientes generaciones.

"Comer todos juntos. Siempre fue importante sentarse a la mesa. Así fueran diez o veinte minutos, sentarnos y hablar. Es allí donde todos nos iniciamos en la gastronomía, con la abuela, con el padre, con la madre, o con quien cada uno tenga más afinidad. Es hasta el día de hoy que, por ejemplo, le digo a papá; me enseñas a hacer esta comida? y lo hacemos juntos y es muy lindo compartir eso, algo que sabemos los dos y que nos gusta. Yo, cuando cocino algo nuevo acá en el colegio, voy a mi casa y les cuento cómo la hice, y quizás es una comida que ellos saben hacer y yo les enseño a hacerla de otra forma. También le enseño a cocinar a mi hijo y así la forma de cocinar se transmite, sigue estando; siempre está el famoso guiso de la abuela o la sopa de mamá, por ejemplo". (Entrevista $\mathrm{N}^{\circ} 12,2016$ )

\section{Innovación en la cocina}

Desde los primeros encuentros estuvo presente el tema de la innovación en la cocina, entendida como la introducción de nuevas técnicas culinarias, materias primas, utensilios y maquinarias para la elaboración de platos, diferenciándose de la cocina tradicional por salir de lo común, de lo ya conocido. Se destacó la oportunidad que brinda la cocina para imaginar y crear apelando a la originalidad a la hora de combinar sabores, técnicas, ingredientes, texturas y colores. También se reflexionó sobre la necesidad de un equilibro entre la innovación y la cocina tradicional y acerca de la relación entre el comensal y la innovación culinaria.

"Es bueno que el comensal sea abierto a probar cosas. Depende de cada uno y de cómo uno quiera adaptarse, uno tiene una cultura y una costumbre, puede probar cosas nuevas o quedarse con lo tradicional. (...) Nosotros una vez hicimos un postre con tomate, salimos de lo común, de lo tradicional, y fue raro. Pero depende de cómo lo tome la gente."

(Entrevista $\left.\mathrm{N}^{\circ} 1,2016\right)$

\section{Aprendizaje en la cocina}

En las entrevistas surgió fuertemente el tema del aprendizaje sobre cocina a través de la observación en diferentes escenarios como la cocina del hogar, los cocineros en TV e Internet. 
Se visualizó a la EET como un espacio para perfeccionar los conocimientos y aprender otros, ejercitar la opinión colectiva en las preparaciones gastronómicas, y se valoró el aprendizaje mutuo al compartir conocimientos culinarios entre alumnos y docentes con igualdad y respeto. Asimismo, se enfatizó en la importancia de aprender todo lo relacionado con la higiene personal, de los alimentos y utensilios.

"A los nueve u ocho años le pedí a mi mamá que me enseñara a hacer una torta, la hice en mi casa, quedé muy contenta, y desde ese día seguí haciendo algunas recetas. También mirando los cocineros en televisión e Internet fui aprendiendo, y en el colegio aprendí pautas higiénicas para la cocina." (Entrevista Nº 6, 2017)

\section{Cocina y cultura}

Se hizo hincapié en cómo la cocina y los saberes culinarios que devienen de ella son modificados por las diferentes costumbres y recursos de cada lugar ya que dependen de la disponibilidad de materia prima, de la economía del lugar y del momento histórico. Se describió a la cocina y el saber culinario como hechos culturales que se transmiten de generación en generación dentro de una misma familia, destacando la importancia de conservar esas recetas familiares, transmitirlas y adaptarlas a los gustos, recursos y condiciones particulares de cada región. También se reflexionó sobre el patrón alimentario poco variado de nuestro país y el miedo de los comensales argentinos a probar alimentos "nuevos":

"Dentro de la familia se transmiten las recetas de generación en generación y esto está influido por la cultura de la región; la materia prima que haya y la forma de cocinarla. La cultura también tiene que ver con que la gente está acostumbrada a comer milanesa, pizza, asado, pollo, etc., y algo distinto o de otro país le genera un cierto rechazo. Acá no". (Entrevista N 10, 2017

\section{Aportes cuantitativos del análisis de las encuestas}

Como explicamos anteriormente, a fin de triangular los resultados de las entrevistas se operacionalizaron algunas de las categorías recurrentes para medirlas en una encuesta cuyos resultados se presentan en este apartado.

Puede observarse en la Figura 1 que la mayoría de los estudiantes de Gastronomía manifestó haber originado su interés por la cocina en el seno familiar. La escuela surgió también como un escenario importante de origen de ese interés.

En tanto, es importante mencionar que, cuando se preguntó a los alumnos si podían identificar un momento puntual en el que surgió su interés por la cocina, el 43 \% respondió que sí.

Respecto de la innovación en la cocina, se les pidió que establecieran un orden de importancia para cuatro frases relacionadas, según su criterio, a la hora de pensar en la innovación en la cocina. Los resultados se presentan en la Figura 2.

Como se observa, la mayoría de los estudiantes otorga mayor importancia a "Combinar sabores, técnicas, ingredientes, texturas y colores" cuando piensa en innovación en la cocina, y menos importancia a "Lograr un equilibrio entre innovación y la cocina tradicional". 
Figura 1. Distribución de la población según origen del interés por la cocina.

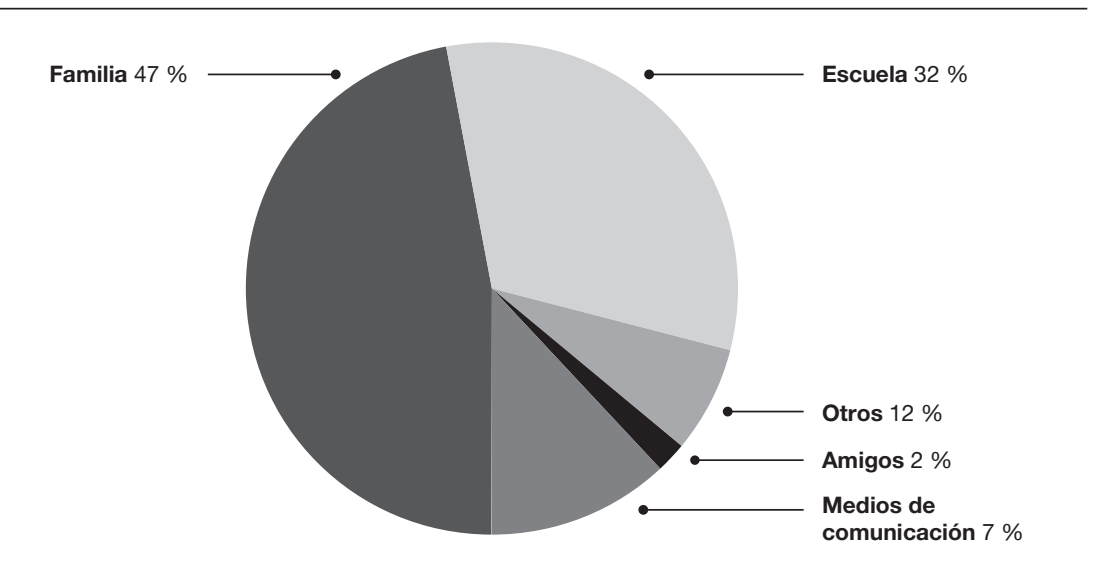

Fuente: Elaboración propia.

Figura 2. Grado de importancia que atribuyen a cada expresión con relación a la innovación en la cocina.

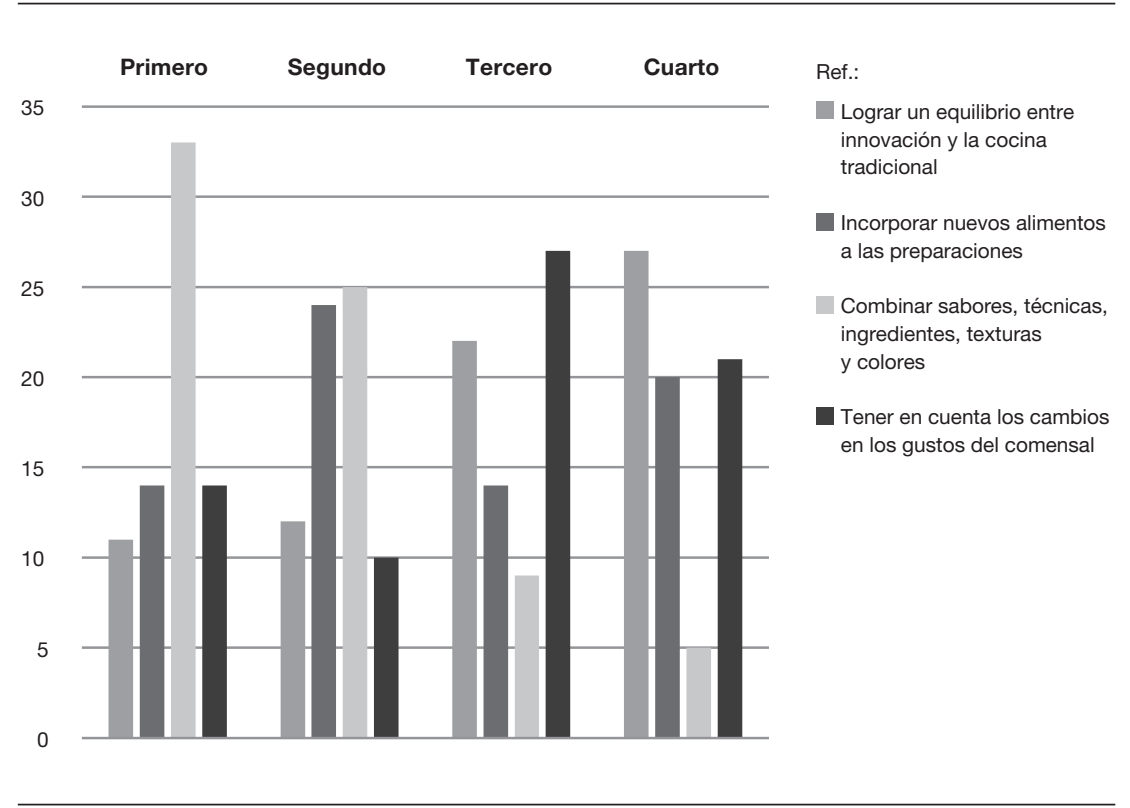

Fuente: Elaboración propia.

El gráfico que sigue nos muestra los resultados obtenidos al preguntar a los estudiantes de Gastronomía cuál de tres aspectos les importaba más en cuanto al nexo entre familia y cocina.

En esta figura se puede ver que la transmisión de saberes culinarios por generaciones y el compartir el acto de comer fueron los aspectos considerados más importantes al pensar en el nexo entre familia y cocina. 
Figura 3. Distribución de la población según importancia atribuida al nexo entre familia y cultura.

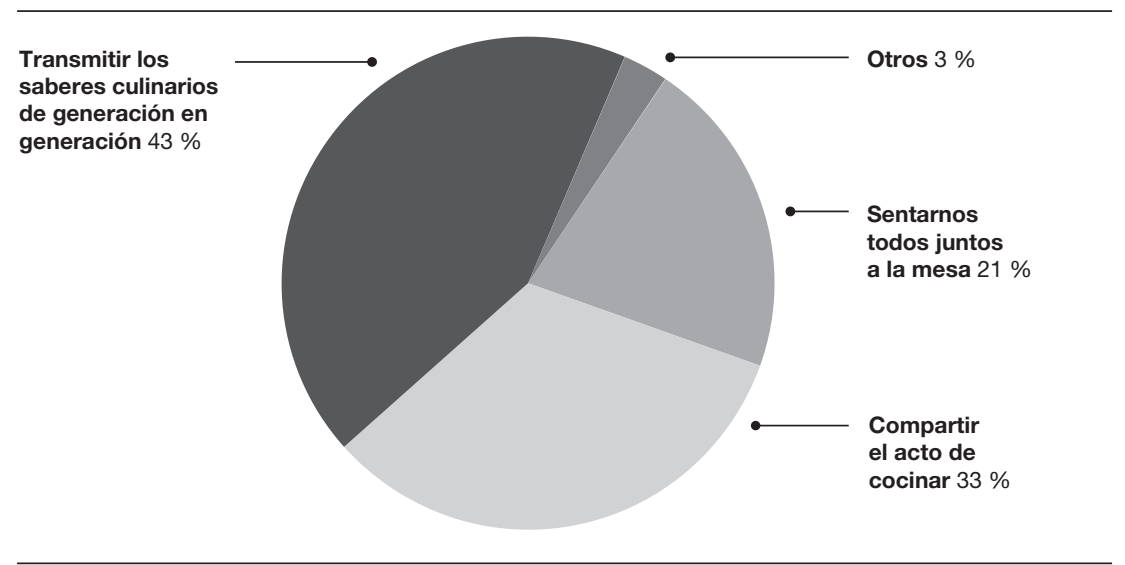

Fuente: Elaboración propia.

Figura 4. Grado de acuerdo con cada una de las expresiones referidas a la cultura y cocina.

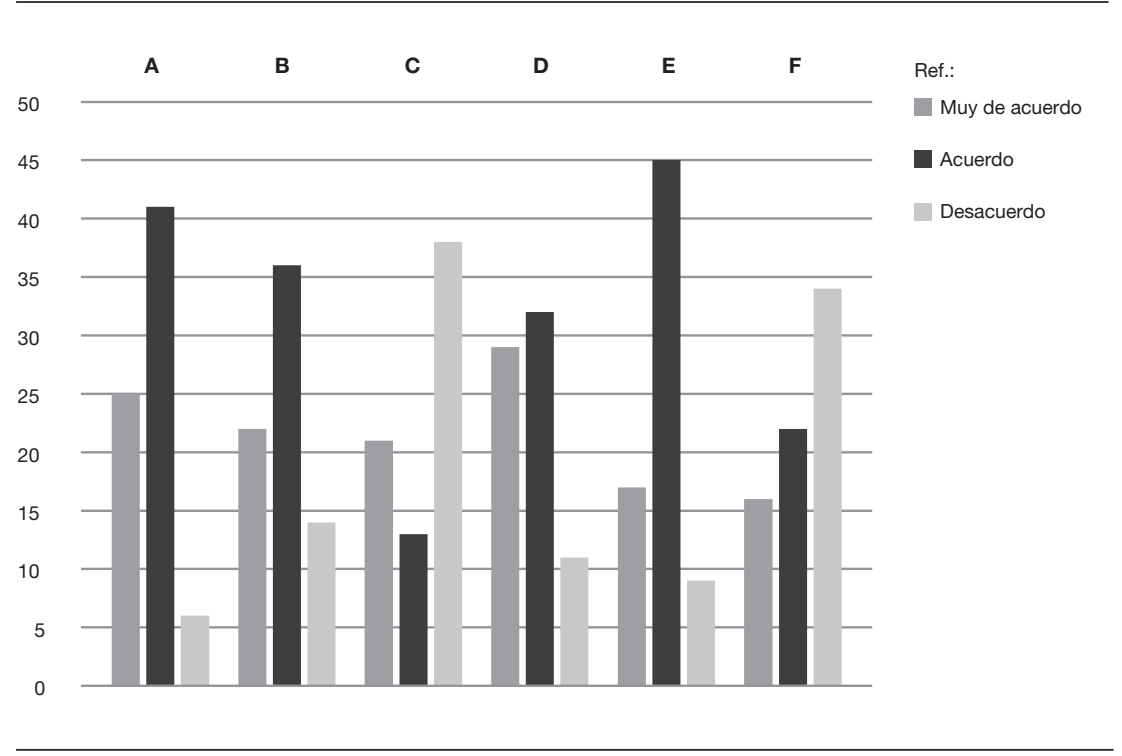

Fuente: Elaboración propia.

Con referecia a la cultura y cocina (Figura 4), se les pidió que indicaran su grado de acuerdo con las siguientes frases:

a) El saber culinario se transmite de generación en generación dentro de una misma familia, permitiendo la continuidad a través del tiempo de diferentes platos.

b) Cuando la cultura está muy arraigada y la gente se acostumbra a los platos de su país es muy difícil incorporar nuevas comidas.

c) En Argentina es todo asado, milanesas, pizza, empanadas... no saben comer otra cosa. 
d) Tener en cuenta la cultura al cocinar es incorporar ingredientes de la región a los platos y adaptarse a los gustos del comensal.

e) La cultura tiene que ver con no perder las preparaciones familiares pero ir incorporando modificaciones que mejoren las recetas.

f) La cocina artesanal.

\section{Aprendizajes de los alumnos de nutrición a partir de la curricularización}

A finales de 2016, primer año de la puesta en marcha del proyecto, se propuso a los grupos de estudiantes de la cátedra de Metodología de Investigación II simular una defensa de lo realizado hasta el momento frente al secretario de Extensión, de tal manera que justificara y fundamentara el financiamiento del mismo durante el segundo año (Clerici, Eckerdt, Monteverde y Naef, 2017).

La decisión de utilizar esta forma de presentación se sustenta con lo propuesto por Prensky (2011), quien considera que los alumnos actuales quieren aprender de manera diferente de los del pasado, utilizando formas que tengan significado para ellos y que les permitan ver el valor que tiene el tiempo que invierten en su educación formal.

Dicha presentación se filmó con el propósito de construir y analizar un registro que pudiera ser posteriormente utilizado para categorizar el aprendizaje de los estudiantes, lo cual se desarrolla en el siguiente apartado.

\section{Lo esperado y lo vivido}

Uno de los aspectos que se destacaron en las presentaciones de los estudiantes tuvo que ver con la gran diferencia que sintieron entre lo que ellos esperaban y lo que "encontraron" al trabajar con los alumnos de la EET $N^{\circ} 1$. En este sentido, se plantearon diferentes incertidumbres o temores relacionados con:

- Embarcarse en un tipo de investigación que les resultaba novedoso.

- Su desempeño frente a la coordinación de los grupos.

- Poder llevar adelante con éxito la técnica de observación.

- Generar vínculo con el adolescente.

- Que los adolescentes manifestaran desinterés por la tarea.

Ellos reconocen que todas las expectativas (incertidumbres y temores) pudieron resolverse sin dificultad con un fuerte trabajo de organización previa y la guía del equipo docente.

"Comenzamos teniendo cierta incertidumbre hacia lo desconocido, en especial a la Investigación-Acción Participativa. Teníamos cierto temor e inseguridad respecto del vínculo con la población participante, ya que, al ser adolescentes, quizá estarían un poco dispersos o no mostrarían interés. Pero no fue así, y en ese aspecto se superaron nuestras expectativas, los chicos se interesaron, se preocuparon y nos respondieron bien siempre." (Entrevista No 4, 2017)

\section{Los aprendizajes destacados}

Los aprendizajes que los estudiantes destacaron luego de participar en esta propuesta incluyen no solo los contenidos propios de la cátedra, como contenidos y procedimientos de investigación, sino también aprendizajes relacionados con los vínculos con personas e instituciones. 


\section{Aprender a planificar intervenciones}

Los alumnos valorizaron la planificación conjunta de las actividades en el aula, la posibilidad de aportar ideas y de conocer previamente todas las acciones a llevar a cabo y los roles de cada uno en las actividades. También reconocieron la riqueza del análisis de los registros de una actividad como insumo de la siguiente.

"Nos sirvió porque tomamos recaudos y desde el primer momento nos tuvimos que informar y planificar cada una de las actividades; previo a cada una siempre íbamos charlando y organizando qué íbamos a decir, quién iba a hablar, cómo íbamos a actuar, etc."

(Entrevista N 8, 2017)

\section{Aprender a llegar a acuerdos}

Hace referencia a la logística desde los acuerdos previos con miembros de la institución hasta los concertados con el grupo previo a la entrada a terreno.

"Desde un principio tuvimos que tener en cuenta muchas cosas; desde las autorizaciones que se solicitan a las instituciones para las visitas, los horarios tanto de la la facultad como del colegio para acordar actividades en conjunto, los horarios de cursada de los chicos de la escuela para acordar día y hora para las entrevistas, etc." (Entrevista N 5, 2016)

\section{Aprender a obtener datos}

Los aprendizajes referidos a las técnicas de obtención de datos que mencionan tienen que ver con la realización de entrevistas y observaciones.

"Respecto a los aprendizajes que adquirimos fueron varios, por ejemplo, la coordinación de un grupo de estudiantes, las técnicas para entrevistar y observar, y también la forma de comunicarnos a la hora de las visitas a las instituciones." (Entrevista $N^{\circ} 11,2016$ )

\section{Aprender a trabajar con grupos}

Los estudiantes valoran como aprendizaje la estrategia al momento de vincularse con los adolescentes, destacando la empatía, el manejo de grupo, la importancia de prestarles atención y darle valor a su opinión.

"Los aprendizajes que tuvimos fueron lograr empatía, coordinar grupos, saber escucharlos con atención. Creo que eso también, fue muy importante para ellos." (Entrevista $\left.\mathrm{N}^{\circ} 2,2017\right)$

\section{Aprender a reconocer los propios aprendizajes}

Se refiere a los aprendizajes que más destacan los estudiantes respecto de los conceptos adquiridos de las lógicas de investigación y las características propias que asumen los elementos del diseño en los diferentes modos de hacer ciencia.

"A lo largo de estos dos últimos años, a través de la cátedra de Metodología de la Investigación aprendimos de las diferentes lógicas que puede tomar una investigación Este año nos enfocamos en la lógica cualitativa y la Investigación-Acción Participativa, mientras avanzábamos en las diferentes actividades del proyecto." (Entrevista N 9, 2016) 
3. Valoración global de la propuesta

Los estudiantes describieron de manera positiva la experiencia llevada a cabo en el marco del proyecto debido a que se sintieron cómodos por haber alcanzado los objetivos propuestos por la cátedra. Además, pusieron de relieve la buena predisposición de los estudiantes del colegio. Consideraron que pudieron realizar todas las actividades grupales asignadas y que en el proceso adquirieron herramientas que les servirán a futuro para su práctica profesional.

"Nos sentimos muy cómodas durante todo el proyecto, tanto la facultad como la escuela nos trataron siempre muy bien. Nos pareció un trabajo beneficioso porque se cumplieron los objetivos acordados por la cátedra, pudimos realizar las actividades que todos teníamos como grupo y, personalmente y como grupo, nos sentimos muy bien desde el primer encuentro. Además los participantes del colegio siempre tuvieron una buena predisposición al realizar las actividades. Estamos muy contentas porque todo funcionó muy bien. El proyecto nos brindó una experiencia muy enriquecedora que nos sirve para un futuro, para la tesis, para todo en realidad." (Entrevista $N^{\circ} 7,2017$ )

\section{Valoración del equipo de cátedra}

El hecho de plantear una propuesta innovadora generó el desafío que implicó, por un lado, modificar el programa de cátedra propuesto, el énfasis en los temas y, por el otro, desarrollar las clases con mayor flexibilidad, adaptándose a las necesidades del proyecto, donde cada semana la clase se planificaba según la actividad o el momento del proyecto. Además, significó un trabajo extra de la logística para establecer el vínculo institucional.

Al estar la propuesta de cátedra en el marco de un proyecto financiado por la UNER, se produjo una reestructuración en función de los requisitos de la convocatoria.

También se evidenció que los estudiantes no solo aprendieron los contenidos y estrategias metodológicas sino todo el proceso para llevar a cabo observaciones, entrevistas y encuestas, el acercarse al otro y saber escuchar. Esto indica que el trabajo realizado les permitió a los estudiantes vivenciar la "cocina de la investigación".

La experiencia posibilitó generar otro vínculo con los estudiantes, verlos desde otro lugar, compartir de manera distinta. Y se resaltó la motivación de los alumnos para realizar las actividades y resolver las situaciones que se iban presentando.

\section{Futuras actividades: sesiones de retroalimentación y generación de una innovación}

Al finalizar el análisis de los registros de observación de los talleres de gastronomía y de la intervención para el análisis de la composición nutricional de las preparaciones, se espera que los estudiantes de 2do. y 3er. año de la Licenciatura en Nutrición puedan poner en discusión estos resultados obtenidos a partir de los diversos abordajes, en una instancia participativa de retroalimentación.

Estamos pensando en llevar a cabo un gran "encontrón"6 donde compartir los resultados elaborados hasta el momento y organizar la estrategia de construcción de menúes que incluyan la valoración nutricional de los platos como producto final del proyecto para ser propuestos a diferentes casas de comida de la ciudad.

6) Este término es tomado por la Dra. Sirvent como traducción del portugués encontrao, para referirse a las sesiones de retroalimentación donde asisten todos los participantes del proyecto. 
Compartimos con la Dra. Sirvent la idea de que las sesiones de retroalimentación no son una "devolución" sino un espacio de construcción colectiva de conocimiento donde el equipo de investigación comparte y pone a consideración de los investigados la marcha del proceso de investigación y sus resultados.

Por ser una instancia grupal en la que se ponen en juego estrategias didácticas, se conjugan investigación, participación y aprendizajes (mutuos) y se considera una estrategia de triangulación in situ.

Uno de los objetivos es generar una instancia de objetivación de la realidad para los alumnos de la EET, entendiendo por objetivación, como se dijo, al proceso clave de reflexión crítica, de aprendizaje y de construcción de conocimiento a través del cual la realidad cotidiana de una población "objeto de estudio" se transforma a su vez en objeto de análisis y de investigación por parte de la misma.

Por otro lado, nos abrirá la posibilidad de enriquecer el conocimiento de los nexos entre gastronomía y nutrición al exponer a su consideración los resultados a los que se arribó.

Se apuesta a la construcción paulatina de maneras diferentes de ver la realidad en el interjuego del conocimiento cotidiano con el conocimiento científico.

Entendemos que de esta forma se favorecerá la apropiación del conocimiento colectivamente construido para que sirva de instrumento para la organización de acciones de transformación de la realidad cotidiana, una de las cuales consistirá en la innovación que implicará la construcción de un menú que ponga en juego todos estos aprendizajes compartidos.

\section{A modo de conclusión}

Este proyecto de curricularización de la extensión se basa en una propuesta didáctica fundamentada que concibe la relación teoría y práctica como eje de los procesos de enseñanza y de aprendizaje. Para los docentes participantes, implica problematizar la práctica y poner a prueba otras formas de enseñar, aprender y evaluar, orientadas a fortalecer y estimular el desarrollo y la formación integral de los futuros profesionales, de manera de crear un espacio donde los contenidos curriculares específicos se resignifiquen desde el oficio de investigador.

Para los estudiantes, supone un aprendizaje no solo de los contenidos y estrategias metodológicas de investigación sino del encuentro con la comunidad: acercarse al otro, saber escuchar, generar conocimiento colectivo, visualizar el compromiso social universitario y el impacto que tienen sus acciones en la comunidad.

Por último, por estar en el marco de una investigación, permite la construcción de categorías teóricas que explican los nexos entre gastronomía y nutrición, que es el objetivo principal del proyecto.

\section{Referencias bibliográficas}

Arocena, R. (2011). Prólogo. Curricularización de la extensión: ¿por qué, cuál, cómo? En Comisión Sectorial de Extensión y Actividades en el Medio (CSEAM). Cuadernos de Extensión № 1. Integralidad: tensiones y perspectivas. Montevideo: Cuadernos Extensión Universidad de la República. Recuperado de http://pim.udelar. edu.uy/publicacion_generica/integralidad-tensiones-y-perspectivas/ 
Camilloni, A. (2018). El desarrollo de las multialfabetizaciones en las experiencias de extensión. Revista $+E$, 7(7), 60-67. Santa Fe, Argentina: Ediciones UNL. doi: 10.14409/extension.v0i7.7051

Castro, J. (2015). Breve repaso sobre la última década en materia de extensión. En Castro, J. y Oyarbide, F. (Comps.). Los caminos de la extensión en la universidad argentina (pp. 19-29). Santa Rosa: Universidad Nacional de La Pampa.

Clerici, C., Eckerdt, C.; Monteverde, A.C. y Naef, E. (agosto de 2017). La investigación-acción-participativa: espacio para aprender del otro y con el otro. Ponencia presentada en las Jornadas de innovación y práctica docente. Entre Ríos, Argentina.

De Michele, D. y Giacomino, M. (2015). Curricularización de la Extensión Universitaria. Primeras experiencias en la UNER. En Castro, J. y Oyarbide, F. (Comps.). Los caminos de la extensión en la universidad argentina (pp. 97-117). Santa Rosa: Universidad Nacional de La Pampa.

Glaser, B. (1992). Basics of Grounded Theory Analysis. Mill Valley, Ca: Sociology Press.

Glaser, B. y Strauss, A. (1967). The discovery of grounded theory: strategies for qualitative research. New York: Aldine Publishing Company.

Giddens, A (1995). La constitución de la sociedad: Bases para la teoría de la estructuración. Buenos Aires: Amorrortu.

Levy, P. (2007). La Cibercultura: Informe al Consejo de Europa. México: Anthropos.

Loustaunau, G. y Rivero, A. (2016). Desafíos de la curricularización de la extensión universitaria. Masquedós, $1(1), 37-45$.

Lucarelli, E. (2009). Teoría y práctica en la universidad. La innovación en las aulas. Buenos Aires: Miño y Dávila. Menéndez, G. (1997) Sistemas de Proyectos de Extensión. Una experiencia de la Universidad Nacional de Entre Ríos. Concepción del Uruguay: UNER.

Perkins, D.N. (2001). La persona-más: una visión distribuida del pensamiento y el aprendizaje. En Salomon,

G. (Comp.). Cogniciones distribuidas. Consideraciones psicológicas y educativas (pp. 126-152). Buenos Aires: Amorrortu.

Prensky, M. (2011). Enseñar a nativos digitales. Madrid: Ediciones SM.

Rigal, L. y Sirvent M.T. (2017) Metodología de la Investigación Social y Educativa: Diferentes caminos de producción de conocimiento. Buenos Aires: Miño y Dávila. Manuscrito en vías de revisión.

Sirvent, M.T. (2012). Conferencia: "La extensión universitaria: concepciones y desafíos del hoy".

Sirvent, M.T. y Monteverde, A.C. (2016). Enseñar a investigar en la universidad. Propósitos, Desafíos y Tensiones en la formación metodológica de posgrado: la experiencia de la Maestría en Salud Mental de la Facultad de Trabajo Social de la UNER. En De Riso, S. y Jacquet, M. (Comps.). Formación en Salud Mental: una experiencia de maestría en la Universidad Pública Argentina. Entre Ríos: EDUNER.

Sirvent, M.T. y Rigal, L. (2011). Investigación acción participativa. Un desafío de nuestros tiempos para la construcción de una sociedad democrática. Quito: Proyecto páramo andino.

Vercellino, S. y Del Carmen, J. (abril de 2014). Curricularización de la extensión universitaria: perspectivas, experiencias y desafíos. Ponencia presentada en las III Jornadas de Extensión del Mercosur JEM, Tandil, Argentina. Recuperado de http://www.extension.unicen.edu.ar/web/jem2014/ponencias/ 